Article

\title{
Codebook-Aided DOA Estimation Algorithms for Massive MIMO System
}

\author{
Shufeng $\mathrm{Li}^{1,2,+}$, Hongda $\mathrm{Wu}{ }^{1, *}$ and Libiao Jin ${ }^{1}$ \\ 1 School of Information and Communication Engineering, Communication University of China, \\ Beijing 100024, China; shufeng_2004@163.com (S.L.); libiao@cuc.edu.cn (L.J.) \\ 2 Wolfson School of Mechanical and Manufacturing Engineering, Loughborough University, \\ Leicestershire LE11 3TU, UK \\ * Correspondence: hongda.wu@cuc.edu.cn; Tel.: +86-1312-1708-282 \\ + Presently, the author is a visiting scholar in Loughborough University.
}

Received: 27 November 2018; Accepted: 24 December 2018; Published: 26 December 2018

check for updates

\begin{abstract}
The conventional direction of arrival (DOA) estimation algorithm is not effective with the tremendous complexity due to the large-scale array antennas in a massive multiple-input multiple-output (MIMO) system. A new frame structure for downlink transmission is presented. Then, codebook-aided (C-aided) algorithms are proposed based on this frame structure that can fully exploit the priori information under channel codebook feedback mechanism. An oriented angle range is scoped through the codebook feedback, which is drastically beneficial to reduce computational burden for DOA estimation in massive MIMO systemss. Compared with traditional DOA estimation algorithms, our proposed C-aided algorithms are computationally efficient and meet the demand of future green communication. Simulations show the estimation effectiveness of C-aided algorithms and advantage for decrement of computational cost.
\end{abstract}

Keywords: DOA estimation; channel feedback; codebook; massive MIMO

\section{Introduction}

Massive multiple-input multiple-output (MIMO) is emerging as a promising technology with large-scale antennas in base station (BS) for future fifth generation wireless communication [1-6]. To meet the diverse type of access with different Quality of Service, such as high data rate application or vehicle to everything with ultra low lantency, antenna collaboration and power control of massive MIMO system are inevitable, which are also guarantee green communication [1-3]. It is well-known that massive MIMO can bring significant improvement in energy efficiency and spectral efficiency by using antenna collaboration to focus energy into small regions of space efficiently. The simulation result of reference [4] proves that the energy efficiency of a massive MIMO system is much higher that the current 4G LTE network. Consequently, massive MIMO is considered as one of the support techniques for future green communication, especially for smart cities networks [5,6]. Precise channel state information (CSI), however, has to be furnished to the transmitter in order to enable the beamforming operation. For Frequency Division Duplex (FDD) massive MIMO systems, the cost of conventional channel estimation schemes requiring orthogonal pilots are prohibitively high; hence, codebook channel feedback mechanism is indispensable in massive MIMO [7,8].

Further, localization can be realized by estimating the Direction of Arrival (DOA) of source signal, while an effective DOA estimation algorithm with low computational complexity is crucial to achieve fast localization, especially for fast varying scenarios [9]. However, as for DOA estimation in massive MIMO systems, the computational complexity is prohibited due to the number of antennas being very large, which lowers the effectiveness of communication. Compressed Sensing 
(CS)-based algorithms are proposed to reduce computational complexity [10]. Reference [11] proposed a complex-valued binary iterative hard thresholding algorithm under CS frame work to reduce computational complexity of DOA estimation for massive MIMO system. However, the estimation performance suffer degradation due to finite measurement. Signal subspace algorithm such as multiple signal classification (MUSIC) algorithm has been widely studied [12]. As a kind of grid-based algorithm, the computational complexity of MUSIC algorithm is related to the angle searching step as well as the number of antennas. For DOA estimation in massive MIMO systems, the computational complexity caused by spectral searching expands drastically with array dimension in massive MIMO systems. Several works were carried out to tackle this computational burden. By using reduced-dimension transformation, a reduced-complexity subspace-based method as reduced-complexity MUSIC for monostatic MIMO radar DOA estimation was presented in [13], which can reduce computational complexity slightly. DOA estimation method based on partial spectral search using transform domain for Co-prime Linear Arrays (Co-prime LAs) is present by Sun et al. [14]. Computational complexity decrement presented in [14] is approximately related to the element number of sub-array of Co-prime LAs. In an FDD massive MIMO system, beamforming combined with codebook channel feedback means that the primal DOA estimation needs to be implemented under deterministic angle space. Further, codebook proposed by [15] has proved that the number of feedback bits is much smaller than the number of BS antenna. In [16], angle-based codebook is designed to reduce feedback overhead of a hybrid precoding millimeter wave massive MIMO system, which motivates us to make full use of feedback information to pre-process DOA estimation.

In this paper, we propose Codebook-aided (C-aided) algorithms to reduce computational complexity for DOA estimation in an FDD massive MIMO system, where DOA estimation is achieved by calculating the parameter of path angles of departure (AoDs). We design a new frame structure for downlink transmission and introduce the concept of codebook channel feedback combing with the classic MUSIC algorithm and newfangled convex optimization algorithm. To be more specific, by utilizing the peculiarity that the path AoDs vary much slower than the path gains $[17,18]$, we execute AoD estimation devotedly on the whole range $\boldsymbol{\Phi}$ (Executing on the whole range $\boldsymbol{\Phi}$ devotedly, that is, for MUSIC algorithm executed when uniform linear arrays is equipped at BS, the angle range of spectral searching is $[-\pi / 2, \pi / 2]$.) during the AoD training stage I within any first half frame of transmission. While for the AoD training stage II, instead of searching the total spectral range, we just dedicate to searching deterministic angle range $\overrightarrow{\boldsymbol{\Phi}}$ which is related to the codebook. This kind of methods are called C-aided algorithms. Compared with primal algorithms, the angle range $\overrightarrow{\boldsymbol{\Phi}}$ for algorithms execution under the auxiliary codebook feedback mechanism can help reduce computational complexity prominently. Meanwhile, for C-aided convex optimization algorithm, we can get the global optimal solutions. The main contributions of the paper are summarized as follows:

- New frame structure for downlink transmission: By leveraging the difference between variation of path AoDs and the path gains, we proposed a new frame structure for downlink transmission. (i) We theoretically prove the peculiarity of AoDs variation. (ii) We decouple AoD estimation of one frame into two separated stages. Within the first and the second half of transmission frame, the AoD estimation are performed in AoD training stage I and AoD training stage II respectively due to the property of AoDs variation.

- Low rank matrix recovery based DOA reconstruction: Apart from the classic MUSIC algorithm, we develop DOA reconstruction method based on low rank matrix recovery, which is referred to as convex optimization algorithm in this paper. We introduce the elastic regularization term to transform the covariance matrix reconstruction problem of the received signal into a semi-definite programming (SDP) problem, which can be effectively solved with polynomial-time complexity.

- Codebook-aided algorithms for DOA estimation: By separating the AoDs acquisition under the frame structure, we propose $\mathrm{C}$-aided algorithms to reduce computational complexity, which includes the C-aided MUSIC algorithm and C-aided convex optimization algorithm. During the AoD training stage II, due to the small angle perturbation, we just focus on deterministic angle 
range $\overrightarrow{\boldsymbol{\Phi}}$ rather $\boldsymbol{\Phi}$ with the help of channel codebook feedback and the AoDs obtained at $A o D$ training stage $I$.

The remainder of this paper is organized as follows. Section 2 introduces the data model, AoD estimation problem and codebook channel feedback. Two kinds of C-aided algorithm is presented and the computational complexity is compared in Section 3. In Section 4, simulation results are elaborated to demonstrate the performance of our proposed algorithm. Finally, the conclusion is given in Section 5 .

Notation: Scalar variables are denoted by normal-face letters, while boldface letters denote vectors and matrices; For a given matrix $\mathbf{A}$, superscripts $\mathbf{A}^{\mathbf{T}}, \mathbf{A}^{\mathbf{H}}$ and $\|\mathbf{A}\|_{Y}$ represent transpose, conjugate transpose and the $\ell_{Y}$ norm operation, respectively; $\mathbb{E}[\cdot]$ denotes the expectation; $\mathbf{I}_{N}$ is the $N \times N$ identity matrix. Notation $\operatorname{tr}(\mathbf{A})$ denotes the trace operator of matrix and $\operatorname{vec}(\mathbf{A})$ is the vectorization operation of vector $\mathbf{A}$. Notation $\mathbf{A} \succeq \mathbf{0}$ denotes that the matrix $\mathbf{A}$ is positive semi-definite. Operation $\max (a, b)$ denotes returning the maximum element between $a$ and $b . \operatorname{Re}[\cdot]$ is the real part operator.

\section{System Model}

In this section, we first briefly introduce the massive MIMO downlink channel model, and then design the frame structure for downlink transmission. Finally, the channel feedback procedure is described.

\subsection{Data Model}

In this paper, a single-cell downlink massive MIMO system is considered. A BS-equipped $N_{T}$ antenna with spacing $d$ communicate with $K$ user equipments (UEs). We just consider the primary paths and assume that the classic uniform linear arrays (ULAs) with incidence from $K\left(N_{T} \gg K\right)$ independent resolvable narrowband source signals [1]. While the waveforms received with perfect synchronization is expressed as

$$
\mathbf{x}(\mathrm{t})=\mathrm{g}_{\mathrm{k}} \mathbf{A}\left(\phi_{\mathrm{k}}\right) \mathbf{s}(\mathrm{t})+\mathbf{n}(\mathrm{t}) .
$$

Channel vector of the $\mathrm{k}$-th path is represented as

$$
\mathbf{h}_{\mathbf{k}}=\mathrm{g}_{\mathrm{k}} \mathbf{a}\left(\phi_{\mathrm{k}}\right),
$$

where $g_{\mathrm{k}}$ is path gain of the k-th $\mathrm{UE}, \mathbf{A}\left(\phi_{\mathrm{k}}\right)=\left[\mathbf{a}\left(\phi_{1}\right), \mathbf{a}\left(\phi_{2}\right), \cdots, \mathbf{a}\left(\phi_{K}\right)\right]$ is the steering vector matrix, and the array response vector $\mathbf{a}\left(\phi_{\mathrm{k}}\right) \in \mathbb{C}^{N_{T} \times 1}$ is given as

$$
\mathbf{a}\left(\phi_{\mathrm{k}}\right)=\left[1, e^{-j 2 \pi \sin \phi_{\mathrm{k}} d / \lambda}, \cdots, e^{-j 2 \pi\left(N_{T}-1\right) \sin \phi_{\mathrm{k}} d / \lambda}\right]^{H},
$$

where $\mathbf{s}(\mathrm{t})=\left[\mathbf{s}_{1}(\mathrm{t}), \mathbf{s}_{2}(\mathrm{t}), \cdots \mathbf{s}_{\mathrm{K}}(\mathrm{t})\right]^{\mathrm{T}}$ is the pilot for AoD training of $K$ UEs satisfying $\mathbb{E}[\mathbf{s}]=0$ and $\mathbb{E}\left[|\mathbf{s}|^{2}\right]=1 . \phi_{\mathrm{k}}$ is the k-th path DOA. $\mathbf{n}(\mathbf{t})$ is additive complex Gaussian noise following $\mathcal{C N} \sim$ $\left(0, \sigma_{n}^{2} \mathbf{I}_{N_{T}}\right)$, where $\sigma_{n}^{2}$ is the variance of noise.

\subsection{AoDs Estimation Problem Formation}

By exploiting the difference of variation between path AoDs and path gains, the estimation of path AoDs and path gains can be achieved by different training stages (We just focus on AoD estimation in this paper and assume the channel gain can be achieved perfectly. This method is also useful for channel estimation, where the path AoDs should be converted to corresponding path AoAs/AoDs.). Further, we propose a new frame structure for downlink transmission as illustrated in Figure 1. Specifically, a frame with time duration $T_{\mathrm{f}}$ consists of two stages of AoDs training with length $M_{\mathrm{AI}} T_{\mathrm{S}}$ and $M_{\mathrm{AII}} T_{\mathrm{S}}$, respectively, and following which are multiple slots where each of them includes paths training stage with length $M_{\mathrm{P}} T_{\mathrm{S}}$ and data transmission with length $M_{\mathrm{D}} T_{\mathrm{S}}$. Here, $M_{\mathrm{AI}}$ and $M_{\mathrm{AII}}$ are the number of pilots for AoD training stage I and AoD training stage II, respectively. $M_{\mathrm{P}}$ and $M_{\mathrm{D}}$, 
respectively, are the number of pilots for path gains training and number of data for transmission in each slot. With the designed frame structure, we make the following assumption,

Assumption 1. Within each transmission frame, AoDs vary slow or keep constant.

AoD variation depend on large scale properties of the scattering environment, thus, this assumption is rational $[17,18]$. Theoretical analysis can be seen in Appendix A.

For each frame of transmission, we conduct AoD estimation twice, which is different for us by exploiting the slowly variation of path AoDs. In the first half transmission frame, we obtain AoD estimation by executing algorithm within whole angle range $\boldsymbol{\Phi}$. Nevertheless, the AoDs vary slow or keep constant, there might still have angle perturbation during one transmission frame. Due to the angle perturbation being relatively small, which is related to the displacement distance of UE. For the second half frame of transmission, AoD estimation could be achieved under deterministic angle range $\overrightarrow{\boldsymbol{\Phi}}$ rather $\boldsymbol{\Phi}$, thus, the computational complexity can be reduced.

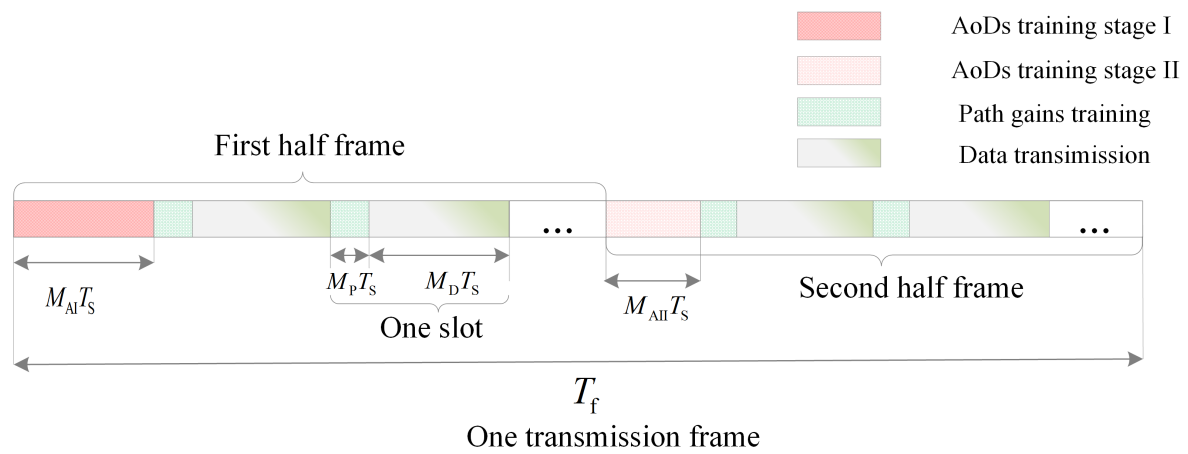

Figure 1. Downlink transmission frame structure consisting of AoD training, path gains training and data transmission.

\subsection{Codebook Channel Feedback}

The channel reciprocity assumed in time division duplexing is not applicable in an FDD protocol. In order to get short-term CSI, i.e., per fading block CSI, and due to the bandwidth constraint over feedback link, CSI must be acquired by measuring reference signals and be conveyed to transmitter with finite bits. While BS receives feedback on desired transmission hypothesis, that is, a precoder vector $\mathbf{w}$ is indicated which is chosen from a set of predefined precoder matrices $\mathbf{W}$ both known at transmitter and receiver. $\mathbf{w}$ and $\mathbf{W}$ also known as codewords and codebook respectively in codebook channel feedback mechanism. Under implicit feedback mechanism, priori information is helpful to form the strongest signal subspace, which means the main channel spatial direction in array signal processing, i.e., angle-based codebook or DFT-based codebook in LTE system [19]. In this paper, we can obtain angular direction matrix by codebook feedback, which is beneficial to orient the approximate angle range $\overrightarrow{\boldsymbol{\Phi}}$. AoD estimation in the AoD training stage II within any frame of transmission could be implemented within $\overrightarrow{\boldsymbol{\Phi}}$ and the computational cost for DOA estimation can be reduced with auxiliary codebook feedback. The corresponding codebook design will be considered in our future work.

\section{DOA Estimation Based on Codebook Feedback}

\subsection{C-Aided MUSIC Algorithm}

In this section, we use classic MUSIC algorithm to achieve DOA estimation. Covariance matrix $\mathbf{R}_{\mathrm{x}}$ with finite $\mathbf{x}(\mathrm{t})$ can be represented as signal- and noise-covariance matrix $\mathbf{U}_{\mathrm{s}}$ and $\mathbf{U}_{\mathrm{n}}$. Eigenvalue $\Lambda_{\mathrm{i}}$ of $\mathbf{R}_{\mathrm{x}}$ satisfy $\Lambda_{1} \geq \Lambda_{2} \geq \cdots \Lambda_{K}>\Lambda_{K+1}=\cdots=\Lambda_{N_{T}}=\sigma_{n}^{2}$, which indicates the number of signal source and $\Lambda_{\mathrm{i}}$ consist of the corresponding subspace. DOA estimation can be obtained through spatial 
spectral searching due to the orthogonality between two subspaces as Equation (4), while $\boldsymbol{\Phi}$ is the angle range for spectral searching.

$$
\begin{aligned}
& \underset{\phi}{\arg \max } \hat{P}_{M U S I C \perp}(\phi)=\frac{1}{\mathbf{a}^{H}(\phi) \mathbf{U}_{\mathrm{n}} \mathbf{U}_{\mathrm{n}}{ }^{H} \mathbf{a}(\phi)}, \\
& \text { s.t. } \phi \in \boldsymbol{\Phi} .
\end{aligned}
$$

In FDD massive MIMO system, the channel vector obtained by parameter estimation $\hat{\mathbf{h}}_{\mathbf{k}}$ during AoD training stage I of the first half frame of transmission is quantized to a quantization vector and then sent to the transmitter, which is realized by codebook $\mathbf{W}=\left[\mathbf{w}_{\mathrm{k}, \mathrm{i}}, \mathrm{i} \in\left\{1,2, \cdots, 2^{\mathrm{B}}\right\}\right]$, where $\mathrm{B}$ is the number of feedback bits and $\mathbf{w}_{\mathrm{k}, \mathrm{i}}$ is codeword which varies with different codebook. The quantization index $Q_{\mathrm{k}}$ for codebook channel feedback is computed as

$$
\begin{aligned}
Q_{\mathrm{k}} & =\underset{\mathrm{i} \in\left[1,2^{\mathrm{B}}\right]}{\arg \min }\left(1-\frac{\left|\hat{\mathbf{h}}_{\mathrm{k}}^{H} \mathbf{w}_{\mathrm{k}, \mathrm{i}}\right|^{2}}{\left\|\hat{\mathbf{h}}_{\mathrm{k}}\right\|_{2}^{2}\left\|\mathbf{w}_{\mathrm{k}, \mathrm{i}}\right\|_{2}^{2}}\right) \\
& =\underset{\mathrm{i} \in\left[1,2^{\mathrm{B}}\right]}{\arg \max }\left|\mathbf{h}^{\prime}{ }_{\mathrm{k}}^{H} \mathbf{w}_{\mathrm{k}, \mathrm{i}}\right|^{2},
\end{aligned}
$$

where $\mathbf{h}^{\prime}{ }_{k}=\frac{\hat{\mathbf{h}}_{\mathrm{k}}}{\left\|\hat{\mathbf{h}}_{\mathrm{k}}\right\|_{2}}$ is the channel direction. $\mathbf{w}_{\mathrm{k}, \mathrm{i}}$ can be fed back to the BS by using B dedicate bits. While after receiving these $B$ channel feedback bits, in other words, the index $Q_{k}$, the BS can generate the fed back channel vector $\tilde{\mathbf{h}}_{\mathrm{k}}=\hat{\mathbf{h}}_{\mathrm{k}} \mathbf{w}_{\mathbf{Q}_{\mathrm{k}}} . \tilde{\mathbf{h}}_{\mathrm{k}}$ obtained in the first half transmission frame contains the information of $\mathbf{a}\left(\phi_{\mathrm{k}}\right)$, since $\mathbf{a}\left(\phi_{\mathrm{k}}\right)$ is completely determined by path AoDs. In the second half frame of transmission, due to the angle perturbation is relatively small, we can execute AoD estimation in the AoD training stage II under deterministic angle range with the help of codebook which helps us steer on specific angle range $\overrightarrow{\boldsymbol{\Phi}}$. The change of $\boldsymbol{\Phi} \rightarrow \overrightarrow{\boldsymbol{\Phi}}$ under this mechanism is beneficial to reduce computational complexity caused by spectral searching.

\subsection{C-Aided Convex Optimization Algorithm}

Supposing the number of array element is larger than the source signal, we get the quantization index $Q_{\mathrm{k}}$ by Equation (5) and obtain the $\overrightarrow{\boldsymbol{\Phi}}$ ulteriorly. We can take the DOA estimation problem as DOA reconstruction based on low rank matrix recovery which is referred as convex optimiazaiont in this paper. Further, by introducing the elastic regularization term, DOA reconstruction can be transformed into SDP problem [20]. Reference [21] is a similar work, but the main idea of handing computational complexity is different.

The covariance matrix $\mathbf{R}_{\mathrm{ss}}$ of noiseless signal is a low rank matrix which satisfies $\operatorname{rank}\left(\mathbf{R}_{\mathrm{ss}}\right)=$ $K \ll N_{T}$. While the problem is modelled as,

$$
\begin{aligned}
& \min _{\mathbf{R}_{\mathrm{ss}}, \sigma_{n}^{2}}\left\|\mathbf{R}_{\mathrm{ss}}\right\|_{0}, \\
& \text { s.t. }\left\|\mathbf{R}_{\mathrm{ss}}-\mathbf{a}\left(\phi_{\mathrm{k}}\right) \mathbf{U}_{\mathrm{s}} \mathbf{a}\left(\phi_{\mathrm{k}}\right)^{H}-\sigma^{2} \mathbf{I}_{N_{T}}\right\|_{2}=0, \\
& \mathbf{R}_{\mathrm{ss}} \geq 0, \sigma_{n}^{2}>0,
\end{aligned}
$$

where two aspects in this model are inextricable, (a) $\ell_{0}$ norm is NP-hard problem, (b) the constrain condition mentioned above is too harsh. The $\ell_{1}$ norm and $\xi$ as error constant related to $\mathbf{R}_{\mathrm{x}}$ are drawn into model.

To augment stability of matrix completion, elastic regularization term $0.5\left\|\mathbf{R}_{\mathrm{ss}}\right\|_{2}^{2}$ is introduced [22], and $\tau$ as equilibrium regularization factor between $\left\|\mathbf{R}_{\mathrm{ss}}\right\|_{1}$ and $0.5\left\|\mathbf{R}_{\mathrm{ss}}\right\|_{2}^{2}$. Moreover, $\left\|\mathbf{R}_{\mathrm{ss}}\right\|_{1}$ can be rewritten as $\operatorname{tr}\left(\boldsymbol{R}_{\mathrm{sS}}\right)$ due to the property of positive semi-definite matrix. An auxiliary optimization 
variable $\varsigma$ is introduced, while the constrain condition changes to $\operatorname{tr}\left(\tau \mathbf{R}_{\mathrm{ss}}+0.5 \mathbf{R}_{\mathrm{sS}}^{H} \mathbf{R}_{\mathrm{ss}}\right) \leq \varsigma$ and the model is described as

$$
\begin{aligned}
& \min _{\mathbf{R}_{\mathrm{ss}, \zeta} \zeta}, \\
& \text { s.t.tr }\left(\tau \mathbf{R}_{\mathrm{ss}}+\frac{1}{2} \mathbf{R}_{\mathrm{ss}}^{H} \mathbf{R}_{\mathrm{ss}}\right) \leq \varsigma, \\
& \left\|\mathbf{J} \operatorname{vec}\left(\mathbf{R}_{\mathrm{ss}}-\mathbf{R}_{\mathrm{x}}\right)\right\|_{2} \leq \xi .
\end{aligned}
$$

The constraint condition in Equation (7) can be rewritten further as

$$
\left\{\begin{array}{c}
\operatorname{tr}\left(\frac{1}{2} \mathbf{R}_{\mathrm{sS}}^{H} \mathbf{R}_{\mathrm{ss}}\right) \leq \varsigma-\operatorname{tr}\left(\tau \mathbf{R}_{\mathrm{ss}}\right), \\
\left\|\mathbf{J} \operatorname{vec}\left(\mathbf{R}_{\mathrm{ss}}-\mathbf{R}_{\mathrm{x}}\right)\right\|^{H}\left\|\mathbf{J} \operatorname{vec}\left(\mathbf{R}_{\mathrm{ss}}-\mathbf{R}_{\mathrm{x}}\right)\right\| \leq \xi^{2} .
\end{array}\right.
$$

Convex optimization problem is turned into standard SDP problem as expressed in Equation (9) [23], and can be effectively solved using the general interior point method with polynomial-time complexity.

$$
\begin{aligned}
& \min _{\mathbf{R}_{\mathrm{ss}, \zeta} \varsigma,} \\
& \text { s.t. }\left[\begin{array}{cc}
2\left(\varsigma-\operatorname{tr}\left(\tau \mathbf{R}_{\mathrm{ss}}\right)\right) & \operatorname{vec}\left(\left(\mathbf{R}_{\mathrm{ss}}\right)\right)^{H} \\
\operatorname{vec}\left(\mathbf{R}_{\mathrm{ss}}\right) & \mathbf{I}_{N_{T}}
\end{array}\right] \succeq 0, \\
& {\left[\begin{array}{cc}
\xi^{2} & \left(\mathbf{J v e c}\left(\mathbf{R}_{s s}-\mathbf{R}_{\mathbf{x}}\right)\right)^{H} \\
\mathbf{J} \operatorname{vec}\left(\mathbf{R}_{\mathrm{ss}}-\mathbf{R}_{\mathbf{x}}\right) & \mathbf{I}_{N_{T}}
\end{array}\right] \succeq 0,}
\end{aligned}
$$

where $\mathbf{J}$ is a selection matrix with size $N_{T}\left(N_{T}-1\right) \times N_{T}{ }^{2}$.

\subsection{Computational Cost Comparison}

The computational cost of MUSIC mainly depends on spectral searching. Complexity of classical MUSIC for ULAs is $\mathcal{O}\left[\boldsymbol{\Phi}\left(2 N_{T}^{2}+N_{T}-N_{T} K\right) / \lambda\right]$ [12], where $\lambda$ is searching step in MUSIC. The decrement of complexity is different from [14], which is connected with the sub-array's element number of Co-prime LA, that is $\max (1 / M, 1 / N)$. The decrement of our proposed C-aided MUSIC is related to the angle shrinking rate $\overrightarrow{\boldsymbol{\Phi}} / \boldsymbol{\Phi}$, which is more flexible than [14]. As for the convex optimization algorithm, the computational complexity mainly depends on SDP which is $\mathcal{O}\left[\left(n_{s d p}^{0.5}\left(m_{s d p} n_{s d p}^{2}+m_{s d p}^{2} n_{s d p}^{2}+m_{s d p}^{3}\right)\right)\right]$, where $n_{s d p}$ is the dimension of semidefinite cone, and $m_{s d p}$ is the number of constraint condition. Codebook feedback is beneficial to diminish $n_{s d p}$ in SDP, thus, reducing the computational cost in convex optimization.

\section{Simulation Results}

Simulations are carried out based on the data model and the proposed C-aided algorithms to investigate DOA estimation performance and computational complexity. In the simulation, we just consider the primary paths of different UEs and assume that the far-field narrowband signal sources are independent. The common simulation parameters are listed in Table 1. 
Table 1. Simulation parameters.

\begin{tabular}{cc}
\hline Parameter Name & Value \\
\hline Array model at BS $N_{T}$ & ULAs \\
Number of BS antennas $N_{T}$ & 32 \\
Number of UEs $K$ & 5 \\
Channel model & AWGN \\
Whole angle range $\boldsymbol{\Phi}$ & {$\left[1.31^{\circ} 2.81^{\circ} 4.51^{\circ} 13.71^{\circ} 17.91^{\circ}\right]$} \\
Direction of independent narrowband signal & {$[-\pi / 2, \pi / 2]$} \\
Angle range $\overrightarrow{\boldsymbol{\Phi}}$ with auxiliary codebook & {$\left[0^{\circ}, 20^{\circ}\right]$} \\
Searching step $\lambda$ for MUSIC & $0.005^{\circ}$ \\
Error constant $\xi$ & 5 \\
Equilibrium regularization factor $\tau$ & 60 \\
Number of pilot for AoD training stage $M_{\mathrm{AI}}, M_{\mathrm{AII}}$ & 500 \\
Number of Monte Carlo simulations $L$ & 300 \\
\hline
\end{tabular}

Figure 2 depicts the spatial spectra of proposed algorithms when the number of pilot for AoD training stage $M_{\mathrm{AI}}=M_{\mathrm{AII}}=500$ and Signal-to Noise Ration (SNR) is $20 \mathrm{~dB}$. As we mentioned in Section 3.1, with the assistance of $Q_{\mathrm{k}}$ and $\mathbf{W}$, we assume that angle range for processing is changed from $\boldsymbol{\Phi}$ to $\overrightarrow{\boldsymbol{\Phi}} \in\left[0^{\circ}, 20^{\circ}\right]$ by $\mathbf{w}_{\mathrm{k}, \mathrm{i}}$. Figure 2 indicates that the C-aided algorithms can distinguish the source signal effectively while spatial spectra have a narrow main-lobe and low side-lobe.

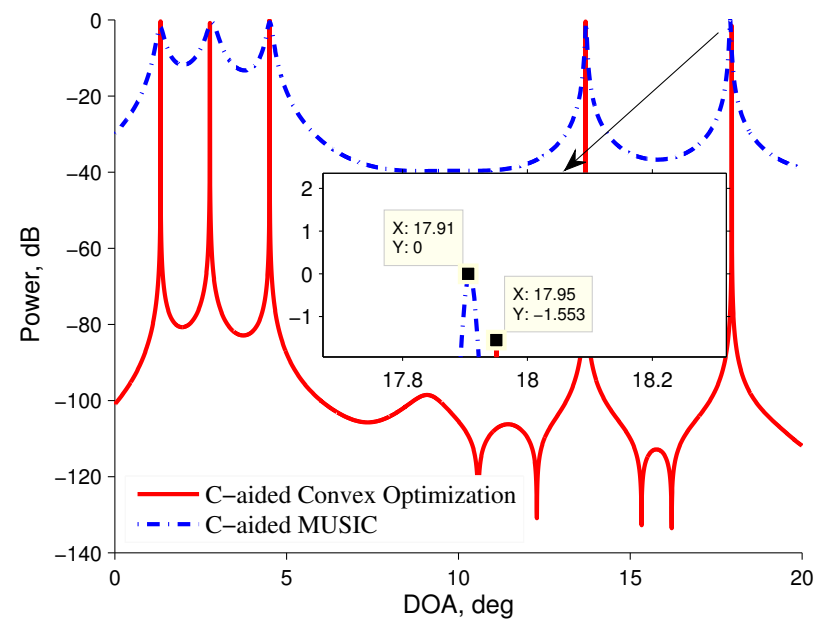

Figure 2. DOA estimation result of proposed algorithms $\left(M_{\mathrm{AI}}=M_{\mathrm{AII}}=500, \mathrm{SNR}=20 \mathrm{~dB}\right)$.

In the next simulation, root mean square error (RMSE) is introduced to measure the DOA estimation performance and is expressed as

$$
R M S E=\sqrt{\frac{1}{L K} \sum_{l=1}^{L} \sum_{\mathrm{k}=1}^{K}\left(\hat{\phi}_{\mathrm{k}}-\phi_{\mathrm{k}}\right)^{2}},
$$

where $L$ is number of Monte Carlo simulations and $\hat{\phi}_{\mathrm{k}}$ is the estimated DOA. We compare the RMSE of different algorithms at different SNRs. To ensure the fairness of computational complexity, sampling points $\Pi$ should be roughly constant, thus, searching step $\lambda$ for primal MUSIC changes from $[\overrightarrow{\boldsymbol{\Phi}} /(\boldsymbol{\Pi}-1)]$ to $[\boldsymbol{\Phi} /(\boldsymbol{\Pi}-1)]$ approximately. For each SNR, 300 Monte Carlo simulations are implemented and Cramer-Rao lower bound (CRLB) as a benchmark is plotted simultaneously [24].

$$
C_{C R L B-M U S I C}(\phi)=\frac{\sigma_{n}^{2}}{2}\left\{\sum_{\mathfrak{t}=1}^{T} \operatorname{Re}\left[\mathbf{s}^{\mathbf{H}}(t) \boldsymbol{\Gamma} \mathbf{s}(\mathbf{t})\right]\right\}^{-1},
$$


where $\boldsymbol{\Gamma}=\mathbf{D}^{\mathrm{H}} \mathbf{U}_{\mathrm{n}} \mathbf{U}_{\mathrm{n}}^{\mathrm{H}} \mathbf{D} . \mathbf{D}=\left[\mathbf{d}\left(\phi_{1}\right), \cdots, \mathbf{d}\left(\phi_{K}\right)\right]$ and $\left.\mathbf{d}\left(\phi_{\mathrm{k}}\right), \mathrm{k} \in\{1,2, \cdots, K\}\right]$ is the first derivative of array response vector $\mathbf{a}\left(\phi_{\mathrm{k}}\right)$. As shown in Figure 3, the accuracies of the proposed C-aided algorithms are slightly better than that of non C-aided algorithm for either MUSIC algorithm [12] or convex optimization algorithm due to the searching step $\lambda$ is smaller with the assist of codebook feedback. As a kind of grid-based algorithm, the proposed C-aided MUSIC algorithm can achieve better estimation performance compared with the corresponding $\mathrm{C}$-aided convex optimization under parameter setting of this paper. However, the accuracy of C-aided convex optimization is not related to the grid, which will outperform C-aided MUSIC algorithm when estimating the off-grid cases. Meanwhile, convex optimization suffers higher computational complexity. Our proposed algorithms can achieve better estimation accuracy and improve the localization precision in future use cases of smart cities. To evaluate the validity of proposed algorithms, computational complexity is simulated in the next experiment.

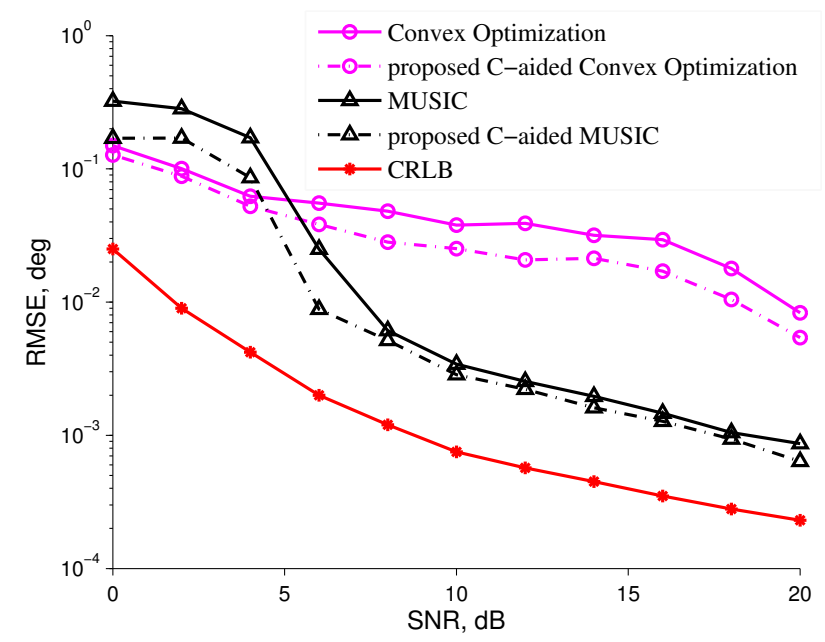

Figure 3. RMSE of DOA estimation algorithms against $\mathrm{SNR}\left(M_{\mathrm{AI}}=M_{\mathrm{AII}}=500\right)$.

In the last simulation, the number of pilots for AoD training is $M_{\mathrm{AI}}=M_{\mathrm{AII}}=500$ and SNR is $20 \mathrm{~dB} .300$ Monte Carlo simulations are implemented at each different antenna number's condition. Under the same hardware implementation, we use the CPU running time of algorithms as an index to reflect the computational complexity. Figure 4 a depicts the running time as the quantitative function of the number of BS antennas to estimate DOAs by MUSIC and proposed C-aided MUSIC. It can be seen that classic MUSIC [12] requires substantially higher complexity due to the whole angle range $\boldsymbol{\Phi}$ under condition of small searching step. Under the parameter setting in Table 1, the computational complexity for classic MUSIC and proposed C-aided MUSIC algorithm are $\mathcal{O}\left(6.68 \times 10^{7}\right)$ and $\mathcal{O}\left(7.42 \times 10^{6}\right)$, respectively, when the number of antennas $N_{T}=36$. In this simulation, compared with MUSIC algorithm, running time saving of proposed C-aided MUSIC is more than $87 \%$ when the number of antennas $N_{T}=36$. Similarly, Figure $4 \mathrm{~b}$ compares the running time between convex optimization algorithm and proposed C-aided convex optimization algorithm. The decrease of $\Pi$ is beneficial to diminish $n_{s d p}$ in SDP under channel codebook feedback, which causes considerable computational burden. With the auxiliary of codebook, the global optimal solutions can be achieved faster. Compared with result presented in Figure $4 \mathrm{a}$, the reduction of computational complexity of the proposed convex optimization algorithm with the help of codebook is more obvious. Clearly, our proposed algorithms aim at oriented angle range rather than whole angle searching and therefore, they impose dramatically lower complexity and can achieve better performance-complexity trade-off. Meanwhile, the proposed C-aided algorithms with lower computational complexity have a great prospect in future communication for smart cities, especially for those applications requiring low latency. 

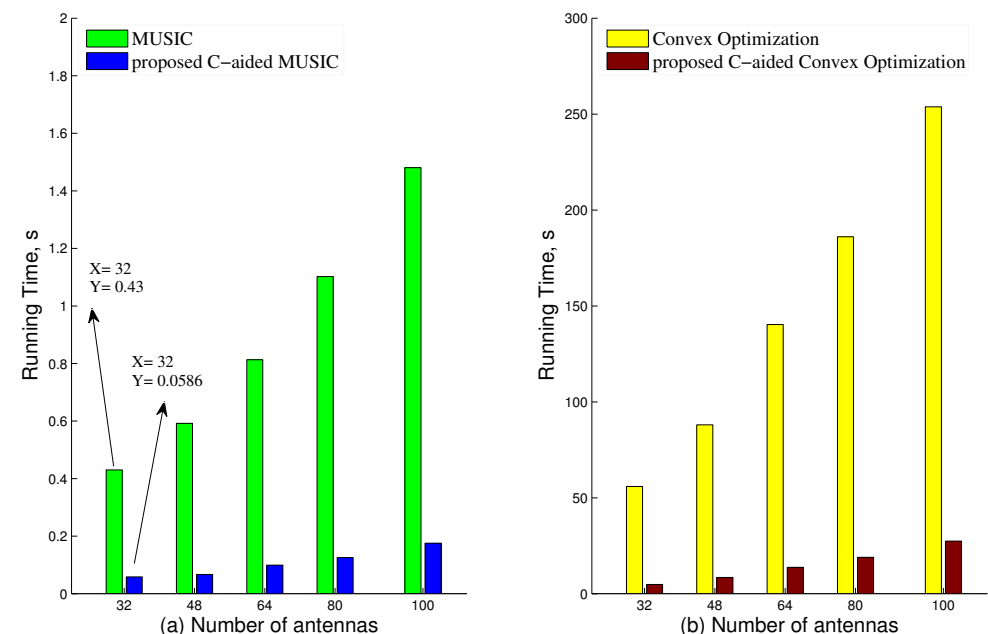

Figure 4. Running time comparison versus different number of antennas $\left(M_{\mathrm{AI}}=M_{\mathrm{AII}}=500, \mathrm{SNR}=20 \mathrm{~dB}\right)$.

\section{Conclusions}

In this paper, we first present a new frame structure for downlink transmission. Based on this frame structure, we propose a codebook channel feedback combined with traditional MUSIC algorithm and newfangled convex optimization algorithm to tackle the computational complexity caused by the increasing number of array in massive MIMO systems. Under a codebook feedback mechanism, we can obtain the oriented angle range, thus, the computational burden of DOA estimation can be reduced notably. Compared with traditional algorithms, our proposed algorithms are computationally efficient and can fit the requirement of future green communication better. Simulation results show the effectiveness of our algorithms.

Author Contributions: Conceptualization, S.L.; Methodology, H.W.; Supervision, L.J.; Writing—original draft, H.W.; Writing-review and editing, S.L.

Funding: This work was supported by the National Natural Science Foundation of China (grant no. 61401407), High-precision Cultivation Project of Communication University of China (grant no. CUC18A006-2) and Engineering Planning Project of Communication University of China (grant no. 2018XNG1851).

Acknowledgments: The authors would like to thank the Editor and the anonymous reviewers for their valuable comments and suggestions.

Conflicts of Interest: The authors declare no conflict of interest.

\section{Abbreviations}

The following abbreviations are used in this manuscript:

$\begin{array}{ll}\text { DOA } & \text { Direction of Arrival } \\ \text { CS } & \text { Compressed Sensing } \\ \text { MIMO } & \text { Multiple Input Multiple Output } \\ \text { BS } & \text { Base Station } \\ \text { CSI } & \text { Channel State Information } \\ \text { FDD } & \text { Frequency Division Duplex } \\ \text { MUSIC } & \text { Multiple Signal Classification } \\ \text { C-aided } & \text { Codebook aided } \\ \text { AoDs } & \text { Angles of Departure } \\ \text { Co-prime LAs } & \text { Co-prime Linear Arrays } \\ \text { UEs } & \text { User Equipments } \\ \text { ULAs } & \text { Uniform Linear Arrays } \\ \text { SDP } & \text { Semi-Definite Programming } \\ \text { RMSE } & \text { Root Mean Square Error } \\ \text { SNR } & \text { Signal-to-Noise Ratio }\end{array}$




\section{Appendix A}

We aim to illustrate the AoDs variation under the assumption. Without loss of generality, we consider one specific path. We assume that there is AoDs variation during one transmission frame, while the variation of AoDs can be expressed as $\left|\theta_{1}-\theta_{2}\right|$, which is denoted by Equation (A1) in the geometric model

$$
\left|\theta_{1}-\theta_{2}\right|=\varepsilon \leq \arctan \left(\frac{S}{D}\right),
$$

where $\theta_{1}$ and $\theta_{2}$ are the AoDs before and after variation; $S$ and $D$ denote the displacement distance of UE and distance between the scatterer and UE, respectively. The equality of Equation (11) holds if $\theta_{1}=\pi / 2$ and we consider two cases in this scenario.

- case 1: $\varepsilon=0$, where the AoDs are constant during one frame of transmission.

- case 2: $\varepsilon$ is small, we have $\varepsilon \approx \tan (\varepsilon)$. When $\left|\theta_{1}-\theta_{2}\right|<\pi / 2 P_{\mathrm{R}}$ with $\pi / P_{\mathrm{R}}$ denoting the resolution of AoDs, we consider the AoDs are relatively constant. As a consequence, we obtain the duration time of one frame $T_{f}$.

$$
T_{f} \leq \frac{\pi D}{2 P_{\mathrm{R}} v}
$$

where $v$ is the average moving speed of UE.

If the resolution of AoDs is set as $0.005^{\circ}$ as mentioned in Section $4, D=100 \mathrm{~m}, v=36 \mathrm{~km} / \mathrm{h}$. The $T_{f}$ is relatively big for the considered parameter, within which the AoDs are almost constant.

\section{References}

1. Björnson, E.; Larsson, E.G.; Marzetta, T.L. Massive MIMO: Ten myths and one critical question. IEEE Commun. Mag. 2016, 54, 114-123. [CrossRef]

2. Rusek, F.; Persson, D.; Lau, B.K.; Larsson, E.G.; Marzetta, T.L.; Edfors, O.; Tufvesson, F. Scaling Up MIMO: Opportunities and Challenges with Very Large Arrays. IEEE Signal Process. Mag. 2013, 30, 40-60. [CrossRef]

3. Hassan, N.; Fernando, X. Massive MIMO wireless networks: An overview. Electronics 2017, 6, 63. [CrossRef]

4. Matalatala, M.; Deruyck, M.; Tanghe, E.; Martens, L.; Joseph, W. Simulations of beamforming performance and energy efficiency for 5G mm-wave cellular networks. In Proceedings of the 2018 IEEE Wireless Communications and Networking Conference (WCNC), Barcelona, Spain, 15-18 April 2018; pp. 1-6. [CrossRef]

5. Gandotra, P.; Jha, R.K.; Jain, S. Green Communication in Next Generation Cellular Networks: A Survey. IEEE Access 2017, 5, 11727-11758. [CrossRef]

6. Mowla, M.M.; Ahmad, I.; Habibi, D.; Phung, Q.V. A Green Communication Model for 5G Systems. IEEE Trans. Green Commun. Netw. 2017, 1, 264-280. [CrossRef]

7. Gao, Z.; Dai, L.; Wang, Z.; Chen, S. Spatially Common Sparsity Based Adaptive Channel Estimation and Feedback for FDD Massive MIMO. IEEE Trans. Signal Process. 2015, 63, 6169-6183. [CrossRef]

8. Shen, W.; Dai, L.; Shi, Y.; Shim, B.; Wang, Z. Joint Channel Training and Feedback for FDD Massive MIMO Systems. IEEE Trans. Veh. Technol. 2016, 65, 8762-8767. [CrossRef]

9. Venieris, E.; Manikas, A. Near-far field multipath spatial-temporal localisation. In Proceedings of the 2017 IEEE International Conference on Communications Workshops (ICC Workshops), Paris, France, 21-25 May 2017; pp. 1049-1054. [CrossRef]

10. Gu, Y.; Zhang, Y.D.; Goodman, N.A. Optimized compressive sensing-based direction-of-arrival estimation in massive MIMO. In Proceedings of the 2017 IEEE International Conference on Acoustics, Speech and Signal Processing (ICASSP), New Orleans, LA, USA, 5-9 March 2017; pp. 3181-3185. [CrossRef]

11. Stöckle, C.; Munir, J.; Mezghani, A.; Nossek, J.A. 1-bit direction of arrival estimation based on Compressed Sensing. In Proceedings of the 2015 IEEE 16th International Workshop on Signal Processing Advances in Wireless Communications (SPAWC), Stockholm, Sweden, 28 June-1 July 2015; pp. 246-250. [CrossRef]

12. Schmidt, R. Multiple emitter location and signal parameter estimation. IEEE Trans. Antennas Propag. 1986, 34, 276-280. [CrossRef] 
13. Zhao, Y.; Shui, P.; Liu, H. Computationally Efficient DOA Estimation for MIMO Radar. In Proceedings of the 2009 2nd International Congress on Image and Signal Processing, Tianjin, China, 17-19 October 2009; pp. 1-3 [CrossRef]

14. Sun, F.; Lan, P.; Gao, B. Partial spectral search-based DOA estimation method for co-prime linear arrays. Electron. Lett. 2015, 51, 2053-2055. [CrossRef]

15. Shen, W.; Dai, L.; Zhang, Y.; Li, J.; Wang, Z. On the Performance of Channel-Statistics-Based Codebook for Massive MIMO Channel Feedback. IEEE Trans. Veh. Technol. 2017, 66, 7553-7557. [CrossRef]

16. Tan, J.; Dai, L.; Li, J.; Jin, S. Angle-based codebook for low-resolution hybrid precoding in millimeter-wave massive MIMO systems. In Proceedings of the 2017 IEEE/CIC International Conference on Communications in China (ICCC), Qingdao, China, 22-24 October 2017; pp. 1-5. [CrossRef]

17. Shen, W.; Dai, L.; Gui, G.; Wang, Z.; Heath, R.W.; Adachi, F. AoD-adaptive subspace codebook for channel feedback in an FDD massive MIMO systems. In Proceedings of the 2017 IEEE International Conference on Communications (ICC), Paris, France, 21-25 May 2017, pp. 1-5. [CrossRef]

18. Chen, Z.; Yang, C. Pilot Decontamination in Wideband Massive MIMO Systems by Exploiting Channel Sparsity. IEEE Trans. Wirel. Commun. 2016, 15, 5087-5100. [CrossRef]

19. Choi, J.; Lee, K.; Love, D.J.; Kim, T.; Heath, R.W. Advanced Limited Feedback Designs for FD-MIMO Using Uniform Planar Arrays. In Proceedings of the 2015 IEEE Global Communications Conference (GLOBECOM), San Diego, CA, USA, 6-10 December 2015; pp. 1-6. [CrossRef]

20. Candes, E.J.; Tao, T. The Power of Convex Relaxation: Near-Optimal Matrix Completion. IEEE Trans. Inf. Theory 2010, 56, 2053-2080. [CrossRef]

21. Zhang, Y.; Zhang, G.; Wang, X. Computationally efficient DOA estimation for monostatic MIMO radar based on covariance matrix reconstruction. Electron. Lett. 2017, 53, 111-113. [CrossRef]

22. Cai, J.F.; Candès, E.J.; Shen, Z. A Singular Value Thresholding Algorithm for Matrix Completion. SIAM J. Optim. 2008, 20, 1956-1982. [CrossRef]

23. Horn, R.A. Topics in Matrix Analysis; Cambridge University Press: Cambridge, UK, 1991.

24. Stoica, P.; Nehorai, A. MUSIC, maximum likelihood and Cramer-Rao bound. IEEE Trans. Acoust. Speech Signal Process. 1989, 37, 720-741. [CrossRef]

(C) 2018 by the authors. Licensee MDPI, Basel, Switzerland. This article is an open access article distributed under the terms and conditions of the Creative Commons Attribution (CC BY) license (http:/ / creativecommons.org/licenses/by/4.0/). 